\title{
$\beta, \beta^{\prime}$-Iminodipropionitrile Impairs Retrograde Axonal Transport
}

\author{
David J. Fink, David Purkiss, and Marina Mata \\ Neurology Research Laboratory, University of Michigan and V.A. Medical Center, Ann Arbor, Michigan, U.S.A.
}

\begin{abstract}
Iminodipropionitrile (IDPN), a neurotoxin, causes redistribution of neurofilaments in axons followed by the development of proximal axonal swellings and, in chronic intoxication, a distal decrease in axonal caliber. The latter changes are caused by a selective impairment in the slow anterograde axonal transport of neurofilament proteins. To assess the role of retrograde axonal transport in IDPN toxicity, we used $\left[{ }^{3} \mathrm{H}\right] N$-succinimidyl propionate ( $\left.\left[{ }^{3} \mathrm{H}\right] \mathrm{NSP}\right)$ to label covalently endogenous axonal proteins in sciatic nerve of the rat and measured the accumulation of radioactively labeled proteins in the cell bodies of motor and sensory neurons over time. IDPN was injected intraneurally $6 \mathrm{~h}$ or intraperitoneally 1 day before subepineurial
\end{abstract}

injection of $\left[{ }^{3} \mathrm{H}\right] \mathrm{NSP}$ into the sciatic nerve, and the animals were killed 1,2 , and 7 days after $\left[{ }^{3} \mathrm{H}\right] \mathrm{NSP}$ injection. Neurotoxicity was assessed by electron microscopic observation of the nerves of similarly treated animals. Both intraneural and intraperitoneal injection of IDPN caused an acute reduction in the amount of labeled proteins transported back to the cell bodies. The early appearance of these changes suggests that alterations in retrograde transport may play a role in the production of the neuropathic changes. Key Words: Axonal transport - Neurotoxicity $-\beta, \beta^{\prime}$ - Iminodipropionitrile-Peripheral nerve. Fink D. J. et al. $\beta, \beta^{\prime}$-Iminodipropionitrile impairs retrograde axonal transport. $J$. Neurochem. 47, 1032-1038 (1986). $\beta, \beta^{\prime}$-Iminodipropionitrile (IDPN) is a neurotoxin whose effect on nerve cells has led to its use as a model for understanding neurofilamentous neuronal pathology. Within $24 \mathrm{~h}$ after subperineurial injection (Griffin et al., 1983) or 4 days after intraperitoneal injection of IDPN (Papasozomenos et al., 1981), the axonal cytoskeleton redistributes as the neurofilaments are displaced toward the periphery of the axon and the microtubules, mitochondria, and smooth endoplasmic reticulum cluster. This is followed over weeks by the development of giant axonal swellings, consisting of masses of disordered neurofilaments, near the nerve cell bodies (Chou and Hartmann, 1964, 1965). In chronically intoxicated animals, the axon distal to the swelling ultimately decreases in caliber. Griffin et al. (1978) have demonstrated that both the proximal axonal swellings and the distal axonal shrinkage result from a specific and selective impairment of the slow anterograde transport of neurofilament proteins.

Anterograde axonal transport serves to supply the axon with the structural and functional macromolecules necessary for continued axonal function. The component of slow axonal transport involving neurofilaments is disrupted in IDPN intoxication, but other components of slow transport and rapid anterograde transport have been reported to be unaffected in the redistribution and accumulation of neurofilaments (Griffin et al., 1978). Retrograde axonal transport from the axon back toward the cell body is generally considered to provide feedback to the cell body about the state of the axon and to play a critical role in maintaining normal neuronal functional integrity. Retrograde transport consists of the "turnaround" transport of anterogradely transported macromolecules and the movement back to the cell body of initially extracellular macromolecules, which are taken up by pinocytosis into specific intraaxonal organelles (Grafstein and Forman, 1980; Bisby, 1981).

Studies of retrograde axonal transport in IDPN intoxication have yielded conflicting results. The accumulation of intramuscularly injected tetanus toxin distal to a ligature on the sciatic nerve appears to be unaffected by IDPN (Griffin et al., 1978). On the other hand, the retrograde transport of intraneurally in-
Received December 27, 1985; accepted March 27, 1986.

Address correspondence and reprint requests to Dr. D. J. Fink at Neurology Research Laboratory, (127), 2215 Fuller Road, Ann Arbor, MI 48105, U.S.A.

A preliminary communication of this work was presented in ab- stract form at the meeting of the American Academy of Neurology in April 1986.

Abbreviations used: DRG, dorsal root ganglia; HRP, horseradish peroxidase; IDPN, $\beta, \beta^{\prime}$-iminodipropionitrile; NSP, $N$-succinimidyl propionate. 
jected horseradish peroxidase (HRP) from the sciatic nerve to the motor neurons of the lumbosacral spinal cord is delayed in both acute and chronic IDPN intoxication (Kuzuhara and Chou, 1981a,b).

Another measure of retrograde axonal transport can be defined using $\left[{ }^{3} \mathrm{H}\right] N$-succinimidyl propionate ( $\left.\left[{ }^{3} \mathrm{H}\right] N S P\right) .\left[{ }^{3} \mathrm{H}\right] N S P$ is an acylating reagent that covalently radioactively labels endogenous axonal proteins in vivo (Fink and Gainer, 1980a,b). After subepineurial injection of $\left[{ }^{3} \mathrm{H}\right] \mathrm{NSP}$ into the sciatic nerve, labeled proteins progressively accumulate in the cell bodies of the sensory and motor neurons that project into the sciatic nerve (Fink et al., 1985a,b, manuscript submitted). The amount of radioactively labeled protein reaching the dorsal root ganglia (DRG) and ventral horn of the spinal cord allows a quantitative estimate of the amount and the rate of transport of endogenous proteins from the axon back to the cell body in sensory and motor neurons, respectively.

To explore further the effect of IDPN on retrograde axonal transport, we have used the $\left[{ }^{3} \mathrm{H}\right] \mathrm{NSP}$ method to determine the amount and rate of retrograde axonal transport in rats following either intraneural or intraperitoneal injection of IDPN. We have compared the time of occurrence of changes in retrograde transport to the time of appearance of the ultrastructural changes characteristic of IDPN neurotoxicity. The results suggest that a reduction in amount of retrograde transport is an early feature of IDPN intoxication.

\section{MATERIALS AND METHODS}

\section{Determination of retrograde transport}

Male Sprague Dawley rats (body weight 200-250 g) were used in all experiments. The animals were anesthetized with chloral hydrate, and the sciatic nerve was exposed in the gluteal region. $\left[{ }^{3} \mathrm{H}\right] \mathrm{NSP}(50 \mathrm{Ci} / \mathrm{mmol}$ in benzene, New England Nuclear) was evaporated to dryness under vacuum on ice and resuspended in $3 \mu \mathrm{l}$ of physiologic saline, $\mathrm{pH}$ 7.4. One microliter was injected subepineurially over a 5 -min period using a 31-gauge needle attached to a Hamilton syringe in a Harvard infusion pump, and the wound was closed with wound clips.

At selected times after injection (1,2, and 7 days), the animals were again anesthetized with chloral hydrate and were killed by perfusion through the heart with $0.1 \mathrm{M}$ phosphate-buffered saline, $\mathrm{pH}$ 7.4. The ipsilateral sciatic nerve, bilateral L4, L5, and L6 DRG, and the spinal cord were rapidly removed and frozen on dry ice. The nerve was minced and placed in $5 \mathrm{~m} M$ Tris- $\mathrm{HCl}$ saline, $\mathrm{pH}$ 7.5. The DRG from each side were pooled in $50 \mu$ lof the same buffer. Sections $(200 \mu \mathrm{m})$ of the spinal cord through the region of the lumbar enlargement were cut in a cryostat at $-8^{\circ} \mathrm{C}$, and the ipsilateral and contralateral ventral horn regions were removed with a $750-\mu \mathrm{m}$ diameter punch. The samples of nerve, DRG, and ventral horn were sonicated in a Kontes microultrasonic cell disrupter for $1 \mathrm{~min}$. The protein in the samples was precipitated twice with $10 \%$ trichloroacetic acid and washed twice with acetone and twice with ether, and the final pellets were suspended in $50 \mu$ l of sample buffer containing $1 \%$ sodium dodecyl sulfate and $1 \% \beta$-mercaptoethanol. After being heated to $95^{\circ} \mathrm{C}$ for $3 \mathrm{~min}$, duplicate ali- quots were taken for determination of radioactivity in an LKB 1218 beta counter.

The amount of retrograde axonal transport to the motor and sensory neuronal cell bodies was calculated by determining the amount of trichloroacetic acid-precipitable radioactivity (ipsilateral to the injection minus contralateral) in the DRG and ventral horn of spinal cord, respectively, at the specified times after $\left[{ }^{3} \mathrm{H}\right] \mathrm{NSP}$ injection. These results were expressed both as total dpm and as a ratio of cell body counts to the amount of radioactivity remaining in the nerve (see Statistical analysis below).

There were five or six animals in each experimental and control group. The number of counts in the contralateral DRG ranged from 30 to 540 in the controls, from 10 to 740 in the intraperitoneal IDPN animals, and from 0 to 640 in the intraneural IDPN animals. The number of counts in the contralateral spinal cord punch samples ranged from 0 to 230 in the controls, from 0 to 210 in the intraperitoneal IDPN animals, and from 0 to 190 in the intraneural IDPN animals.

\section{IDPN injection}

The effects of both intraperitoneal and intraneural injection of IDPN were assessed. IDPN was diluted 1:1 with phosphate-buffered saline, pH 7.3. For intraneural injection, $5 \mu$ l of IDPN was injected subepineurially into the sciatic nerve using a 31-gauge needle attached to a Hamilton syringe $6 \mathrm{~h}$ before injection with $\left[{ }^{3} \mathrm{H}\right] \mathrm{NSP}$. Control animals for the intraneural experiments were injected in a similar manner with $5 \mu \mathrm{l}$ of saline $6 \mathrm{~h}$ before $\left[{ }^{3} \mathrm{H}\right] \mathrm{NSP}$ injection. In the intraperitoneal group, animals were injected with IDPN, $2 \mathrm{~g} / \mathrm{kg}$ as a single intraperitoneal dose, $24 \mathrm{~h}$ before injection of $\left[{ }^{3} \mathrm{H}\right] \mathrm{NSP}$.

\section{Electron microscopy}

At specific times after intraperitoneal ( 1 and 8 days) or intraneural ( 1 day) injection of IDPN, the dorsal and ventral roots and the sciatic nerve were examined ultrastructurally. Under chloral hydrate anesthesia, the animals were perfused through the heart with $0.1 \mathrm{M}$ phosphate buffer followed by $3 \%$ glutaraldehyde, $0.5 \%$ paraformaldehyde, and

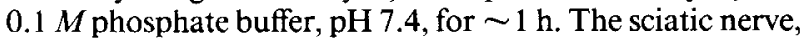
roots, and DRG were dissected out and kept in fixative for an additional $2 \mathrm{~h}$ at $4^{\circ} \mathrm{C}$, postfixed in $2 \% \mathrm{OsO}_{4}$ in the same buffer, dehydrated, and embedded in a Polybed-Araldite mixture. Thin sections were stained with uranyl acetate and examined in a JEOL 100 S electron microscope.

\section{Statistical analysis}

Several analyses were used to compare retrograde transport in control and IDPN-treated animals. Because of the variation in the amount of injected radioactivity, analyses were performed using the ratio of counts in the cell body to counts remaining in the nerve. These ratios were on the order of $10^{-3}-10^{-5}$. Using analysis of covariance, we assessed the relationship between the counts in the cell bodies and those remaining in the nerve in individual control and experimental animals and found there was no statistically significant linear relationship between those counts. We performed analyses of variance on the counts and on the ratios using Bonferroni's procedure for multiple comparisons to control values for the multiple comparisons being made. Results from both analyses led to the same conclusions, but we found that the analyses of counts accounted for a greater proportion of the variability than did the analyses on the ratios. Therefore, we have expressed the data as trichloro- 

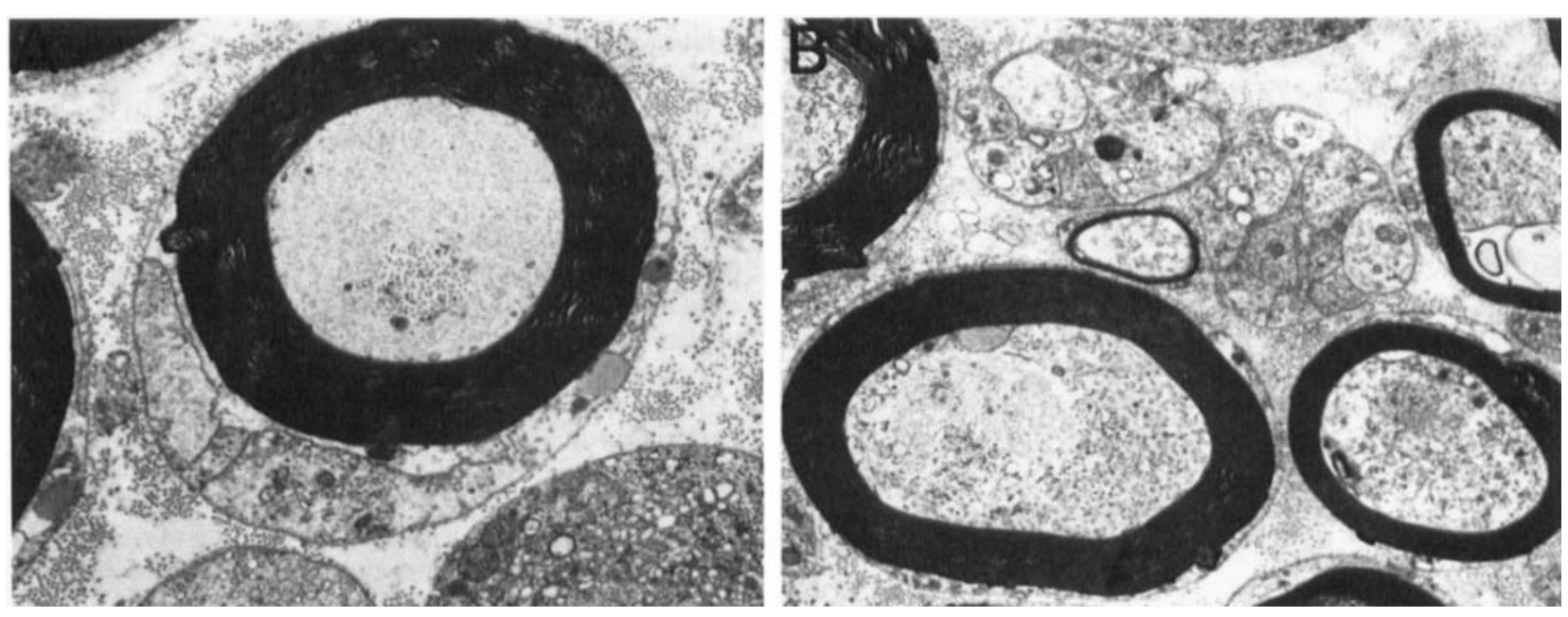

FIG. 1. Sciatic nerve $24 \mathrm{~h}$ after intraneural injection of $5 \mu$ l of IDPN. A: Many fibers showed redistribution of the cytoskeleton with clustering of microtubules centrally and the neurofilaments peripherally. B: Some fibers, however, showed evidence of vesicular accumulation and retraction of the axolemma from the myelin sheath. $A, \times 10,900 ; B, \times 8,400$.

acetic acid-precipitable counts (ipsilateral minus contralateral) rather than as ratios in the figures presented here.

\section{RESULTS}

The normal accumulation of radioactively labeled proteins in the DRG and the spinal cord at 1,2, and 7 days is shown in the control curves of Figs. 5 and 6, respectively. In the DRG, the increase in counts from 1 to 2 days is statistically significant $(p<0.005)$, as is the increase from 2 to 7 days $(\mathrm{p}<0.005)$. In the spinal cord, the accumulated counts are less, and the apparent increase from 1 to 2 days is not statistically significant ( $p=0.6)$, whereas the increase from 2 to 7 days is significant $(p<0.001)$. Beyond 7 days, DRG counts drop significantly ( $p<0.001$ by 14 days), whereas the spinal cord counts plateau at 14 and 21 days and do not drop significantly until 28 days after injection (Fink et al., 1985b, manuscript submitted).

Intraneural injection of IDPN causes redistribution of the axonal cytoskeleton, which begins within hours after the injection, is maximal at $6 \mathrm{~h}$, and then resolves over days (Griffin et al., 1983). In our initial series of experiments, we injected IDPN intraneurally and $6 \mathrm{~h}$ later injected $\left[{ }^{3} \mathrm{H}\right] \mathrm{NSP}$ subepineurially distal to the site of the IDPN injection. Animals were then allowed to survive 1 or 7 days, during which retrograde axonal transport occurred. At 1 day after intraneural IDPN injection, substantial redistribution of neurofilaments, clustering of tubules and mitochondria, and some axonal swelling is apparent (Fig. 1A). In some of the fibers, there is also evidence of early stages of degeneration, manifest by accumulation of vesicular and tubulovesicular profiles (Fig. 1B).

Intraneural injection of saline caused a reduction in the amount of radiolabeled material accumulating at the DRG and spinal cord when compared with uninjected controls. This may be due to nerve damage from elevated endoneurial pressure caused by the relatively large volume $(5 \mu \mathrm{l})$ of the injection. Nonetheless, the increase normally found from 1 to 7 days in labeled proteins at the cell body is still apparent in these saline control animals. Intraneural IDPN caused a significant reduction from the value in saline controls in transport to the DRG after 7 days of transport ( $p<0.0001 ;$ Fig. 2$)$ and in transport to the spinal cord after 7 days of transport ( $p<0.005$; Fig. 3 ). The accumulation in the cell bodies $24 \mathrm{~h}$ after $\left[{ }^{3} \mathrm{H}\right] \mathrm{NSP}$ injection appeared to be reduced in both DRG and

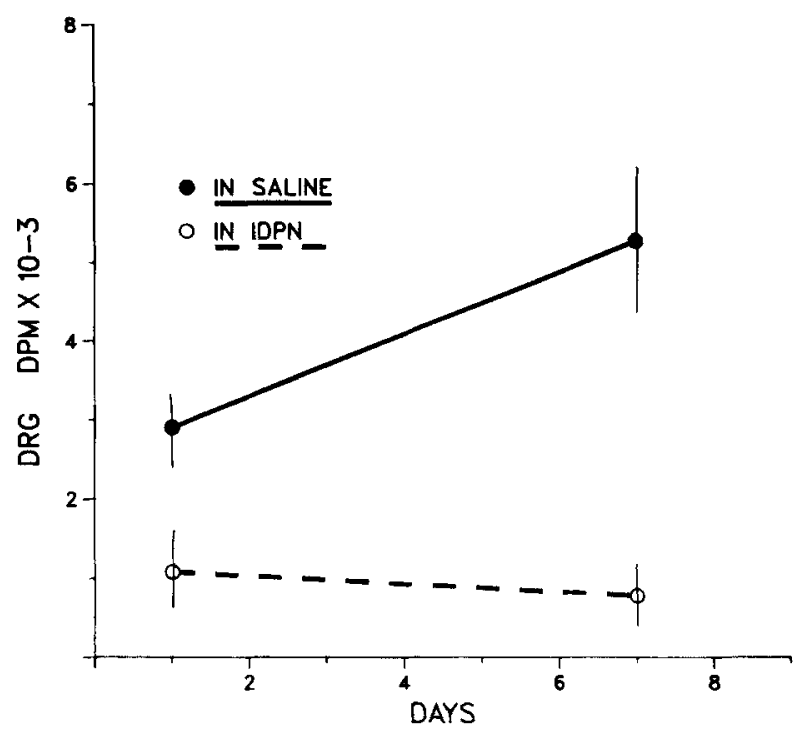

FIG. 2. Retrograde transport of labeled protein from the sciatic nerve to the cell bodies of DRG neurons in animals injected with $\left[{ }^{3} \mathrm{H}\right] N S P$ $6 \mathrm{~h}$ after intraneural injection of either saline or IDPN. Injection of IDPN caused a significant reduction from the saline control value at 7 days $(p<0.005)$ but not at 1 day. Data are mean \pm SEM (bars) values. 


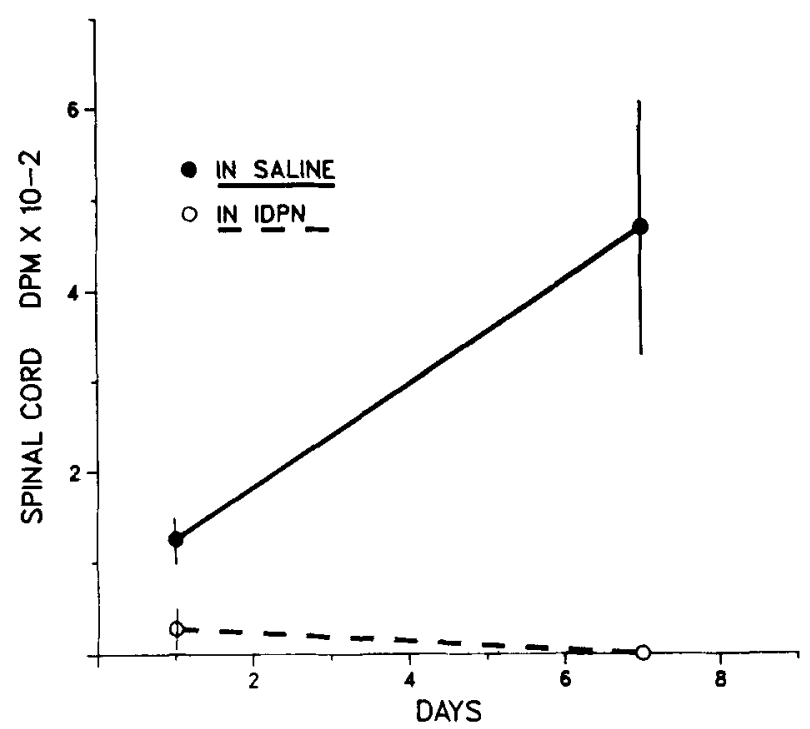

FIG. 3. Retrograde transport of labeled protein from the sciatic nerve to the cell bodies of ventral horn neurons in animals injected with $\left[{ }^{3} \mathrm{H}\right] \mathrm{NSP} 6 \mathrm{~h}$ after intraneural injection of either saline or IDPN. Injection of IDPN caused a significant reduction compared with the saline control value at 7 days $(p<0.02)$ but not at 1 day $(p=0.2)$. Data are mean \pm SEM (bars) values.

spinal cord, but those reductions were not statistically significant (Figs. 2 and 3).

These results suggest that, concurrent with toxininduced cytoskeletal rearrangement, there is disruption of the late accumulation of retrogradely transported radioactive proteins in motor and sensory neurons. To avoid the possible confounding variable introduced by intraneural injection and to determine whether the reduction in retrograde transport occurs before or after the cytoskeletal rearrangement, we went on to study animals after intraperitoneal injection of IDPN.

At 1 day after intraperitoneal injection of IDPN, the nerve, dorsal root, and ventral root all appear normal (Fig. 4A-C). By 8 days after intraperitoneal injection, there is redistribution of microtubules and neurofilaments in the nerve (Fig. 4D), and massive swellings occur in the proximal dorsal and ventral roots (Fig. 4E and F), as have been described above. Despite the fact that the nerve and roots appear normal at 1 day, retrograde transport in the sensory neurons is reduced to $30 \%$ of control values $(p<0.005)$ at 1 day (Fig. 5). There is no significant difference between the results of intraneural or intraperitoneal injection of IDPN with 1 day of transport or at later times. The differences in retrograde transport to the DRG after 2 and 7 days of transport are also statistically significant ( $p$ $<0.005$ ). Transport in motor neurons is affected by intraperitoneal IDPN in a manner similar to that of intraneural injection. There are no significant differences from control values at 1 and 2 days, but a significant reduction in transport occurred at 7 days ( $p$ $<0.006$; Fig. 6).

\section{DISCUSSION}

These studies demonstrate that alterations in retrograde axonal transport occur early in IDPN neurotoxicity. An intraneural injection of IDPN causes cytoskeletal redistribution within hours. By 1 day, not only is redistribution apparent, but early evidence of degeneration in some fibers has begun to appear. Griffin et al. (1983) have suggested that the degenerative changes may be an artifact of the intraneural injection. In that condition, retrograde transport to both the motor and sensory neurons is significantly reduced at 7 days, and although the amount of radiolabeled proteins reaching the cell bodies appears to be reduced at 1 day, that reduction is not statistically significant.

Are these alterations in retrograde transport due to the cytoskeletal disruption, or are they caused by other neurotoxic effects of IDPN? To answer that question, we went on to study animals after intraperitoneal administration of IDPN. At 1 day after intraperitoneal IDPN injection, there are no apparent changes in cytoskeleton or axonal architecture. Despite the absence of ultrastructural changes, transport to the sensory neurons is reduced both at the early ( 1 and 2 days) as well as the late (7 day) intervals, whereas transport to the motor neurons is not affected at the early intervals but is significantly reduced by the late interval.

The effect on the later accumulation of retrogradely transported proteins, which accounts for the majority of labeled transported proteins, is similar in motor and sensory neurons. The reason why retrograde transport to the motor neurons at the earlier intervals appears to be less affected by IDPN is not clear. The number of counts that reach the motor neurons are an order of magnitude less than those reaching the DRG, and the SE is much greater relative to the number of counts, so it is possible that a small but significant difference failed to be detected. Alternately, there may be real differences in the vulnerability of rapid retrograde transport to IDPN between motor and sensory neurons.

Kuzuhara and Chou $(1981 a, b)$ found that, in rats 3 days or 4 weeks after intraperitoneal injection of IDPN, HRP injected into the sciatic nerve was delayed in its arrival at the large neurons in the spinal cord, whereas the labeling of small neurons appeared normal. By $48 \mathrm{~h}$ after HRP injection, they could detect tubules and vesicles containing HRP reaction product densely in the axonal swellings and also in the neuronal somata. Although we were unable to detect a statistically significant decrease in transport to the motor neuron perikarya until a longer time for transport had elapsed, these results are, in general, in agreement with our findings that a defect in retrograde transport to the cell bodies is a characteristic of IDPN neurotoxicity.

In contrast, Griffin et al. (1978) were unable to demonstrate a defect in retrograde axonal transport in rats 

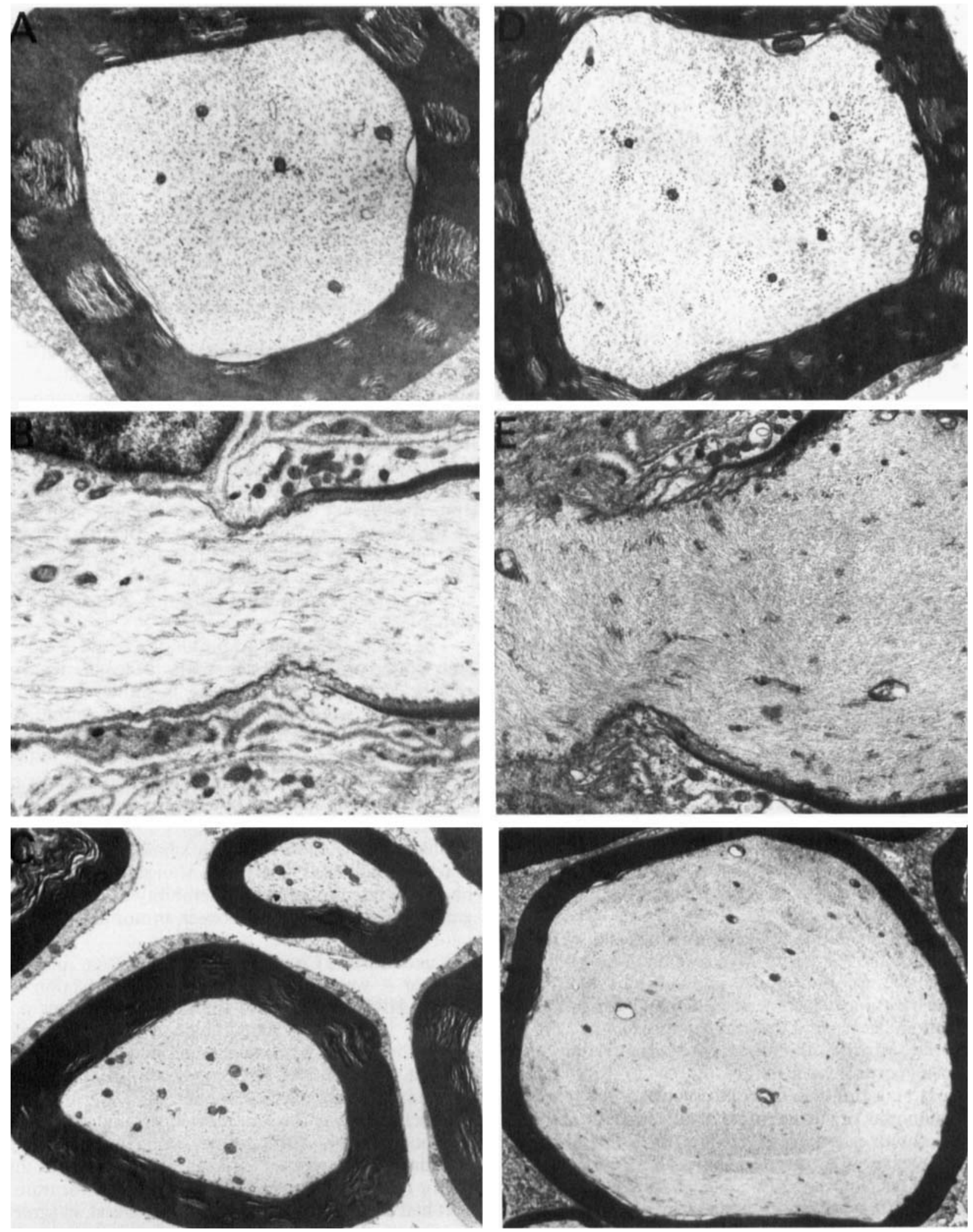

FIG. 4. Comparison of the ultrastructural appearance of nerve, ventral root, and dorsal root at 1 day (A-C) and 8 days (D-F) after intraperitoneal injection of IDPN. At 1 day, the nerve appears normal (A), but by 8 days, clustering of the microtubules into discrete groups within the neurofilament matrix is apparent (D). Similarly, the proximal ventral root appears normal 1 day after IDPN injection (B), but by 8 days, massive swellings created by the accumulation of disordered neurofilaments are found $(E)$. Like the ventral root, the dorsal root appears normal at 1 day (C) but by 8 days is distorted by the accumulation of neurofilaments $(F) . A, \times 7,200 ; B, \times 16,300 ; C, \times 12,200 ; D$, $\times 10,200 ; E, \times 16,300 ; F, \times 12,200$. 


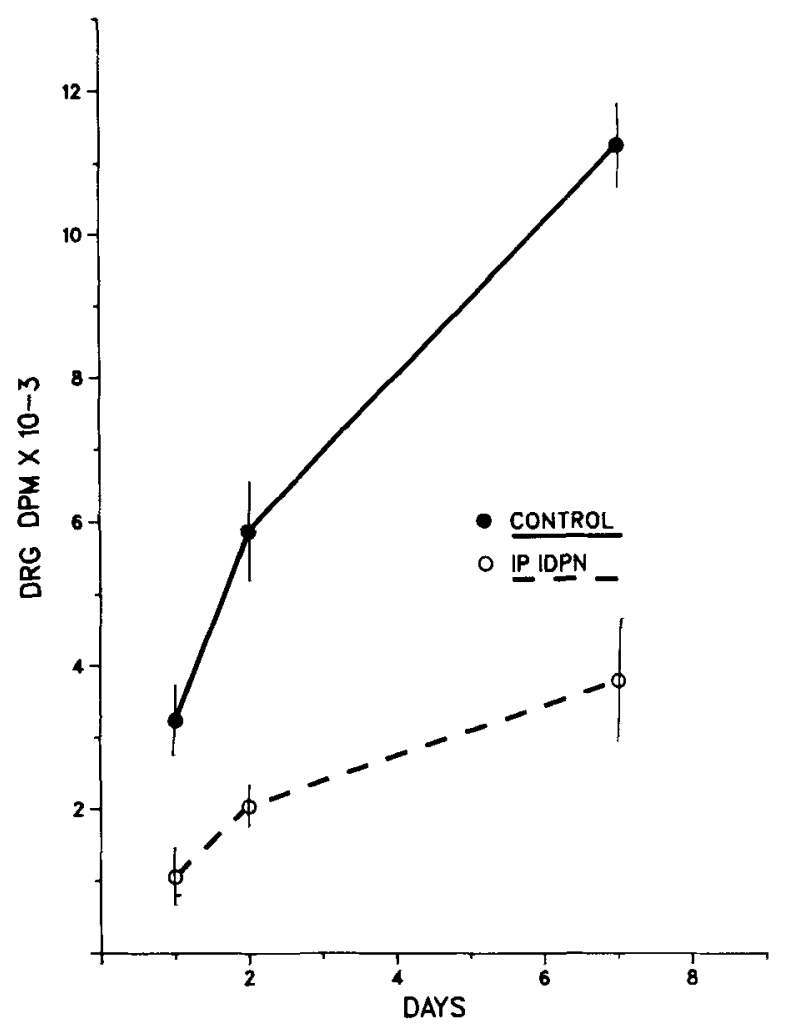

FIG. 5. Retrograde transport of labeled protein from the sciatic nerve to the cell bodies of DRG neurons in animals injected with $\left[{ }^{3} \mathrm{H}\right]$ NSP $24 \mathrm{~h}$ after intraperitoneal injection of IDPN. The accumulation of labeled proteins at the cell bodies is reduced significantly when compared with that in uninjected animals at 1 day $(p$ $<0.005), 2$ days $(p<0.005)$, and 7 days $(p<0.005)$ after $\left[{ }^{3} H\right] N S P$ injection. Data are mean \pm SEM (bars) values.

after long-term IDPN intoxication. They found that, $18 \mathrm{~h}$ after intramuscular injection of ${ }^{125}$ I-labeled tetanus toxin, the amount of radioactivity accumulating distal to a ligature on the sciatic nerve was not significantly reduced. There are two major differences between the paradigm they used for measuring axonal transport and the one used in this study. They measured retrograde transport from the terminal to a point in the axon, whereas we measured transport from the axon to the cell body. It is possible that the disruption of retrograde transport occurs in proximal but not distal axons, so that transport from the terminal to the axon is unaffected whereas transport from the axon to the cell body is impaired. A second possibility - that the retrograde transport of exogenous macromolecules is affected in a manner different from the transport of endogenous axonal proteinscould account for the difference between their results and ours, but would not explain the difference between their results and the results of Kuzuhara and Chou $(1981 a, b)$.

The results of our study - that a decrease in retrograde axonal transport is an early feature of IDPN intoxication-is part of the growing evidence that al- terations in retrograde transport may occur in many toxic neuropathies. Defects in the "turnaround" transport of anterogradely transported radiolabeled proteins have been demonstrated in neuropathies induced by hexacarbons, zinc pyridinethione, and $p$ bromophenylacetylurea (Sahenk and Mendell, 1980, 1981; Jakobsen and Brimijoin, 1981). Acrylamide in doses of $100 \mathrm{mg} / \mathrm{kg}$, insufficient to cause a clinically apparent neuropathy, nonetheless reduces the retrograde "turnaround" transport of labeled proteins in rats (Jakobsen and Sidenius, 1983). Also, single doses of acrylamide have been shown to reduce the velocity of retrograde transport of ${ }^{125}$ I-labeled tetanus toxin by up to $75 \%$ within hours of administration of the toxin (Miller and Spencer, 1984).

The latter two studies have led to speculation that the alterations in retrograde transport may be significant in the chain of events that lead to the neuropathy. It has long been suspected, principally on the basis of the reaction of neurons to axonal injury, that retrogradely transported substances may play a critical role in the continued normal function of the neuron (Cragg, 1970). The alterations in transport that have been demonstrated in the pathologic states noted above and the evidence that these changes may be a

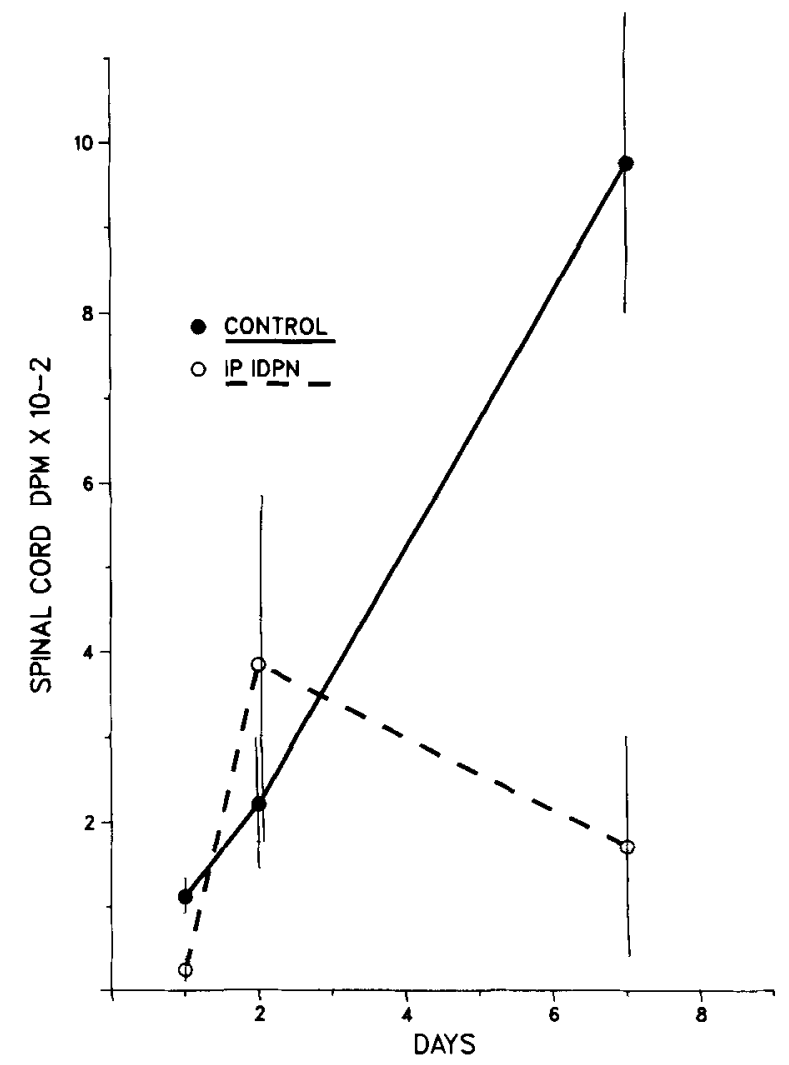

FIG. 6. Retrograde transport of labeled protein from the sciatic nerve to the cell bodies of ventral horn neurons in animals injected with $\left[{ }^{3} \mathrm{H}\right]$ NSP $24 \mathrm{~h}$ after intraperitoneal injection of IDPN. The accumulation of labeled proteins is reduced significantly at 7 days of transport $(p<0.006)$. Data are mean \pm SEM (bars) values. 
very early manifestation of intoxication lend support to that notion. Our findings that reductions in axonal transport are an early and persistent change after single doses of IDPN are further evidence that some of those transported proteins may serve critical signal functions in the biology of the neuron.

Acknowledgment: The statistical analysis was performed by Dr. Judy Bromberg of V.A. HSR\&D. We wish to acknowledge the excellent technical assistance in histology and photography of Julie Staple and Barbara Rogers and the secretarial assistance of Carolyn White. The graphs were produced by Dr. Ron Craig. This research was supported by V.A. Merit Review grants to Dr. Mata and to Dr. Fink.

\section{REFERENCES}

Bisby M. A. (1981) Functions of retrograde axonal transport. Fed. Proc. 41, 2307-2311.

Chou S. H. and Hartmann H. A. (1964) Axonal lesions and waltzing syndrome after IDPN administration in rats. Acta Neuropathol. (Berl.) 3, 428-450.

Chou S. H. and Hartmann H. A. (1965) Electron microscopy of focal neuroaxonal lesions produced by beta,beta'-iminodipropionitrile (IDPN) in rats. Acta Neuropathol. (Berl.) 4, 590-603.

Clark A. W., Griffin J. W., and Price D. L. (1980) The axonal pathology in chronic IDPN intoxication. $J$. Neuropathol. Exp. Neurol. 39, 42-55.

Cragg B. G. (1970) What is the signal for chromatolysis? Brain Res. $23,1-21$

Fink D. J. and Gainer H. (1980a) Retrograde axonal transport of endogenous proteins in sciatic nerve demonstrated by covalent labeling in vivo. Science 208, 303-305.

Fink D. J. and Gainer H. (1980b) Axonal transport of proteins: a new view using in vivo covalent labeling. $J$. Cell Biol. 85, 175186.

Fink D. J., Purkiss D., and Mata M. (1985a) Rapid retrograde transport of proteins in sensory neurons. Brain. Res. 345, 394-397.

Fink D. J., Purkiss D., and Mata M. (1985b) Retrograde axonal transport in motor and sensory neurons of the rat sciatic nerve. (Abstr) Soc. Neurosci. Abstr. $11,1304$.

Grafstein B. and Forman D. (1980) Intracellular transport in neurons. Physiol. Rev. 60, 1167-1283.

Griffin J. W., Hoffman P. N., Clark A. W., Carroll P. T., and Price D. L. (1978) Slow axonal transport of neurofilament proteins: impairment by beta,beta'-iminodipropionitrile administration. Science 202, 633-635.

Griffin J. W., Fahnestock K. E., Price D. L., and Hoffman P. N. (1983) Microtubule-neurofilament segregation produced by beta,beta-iminodipropionitrile: evidence for the association of fast axonal transport with microtubules. J. Neurosci. 3, 557566.

Jakobsen J. and Brimijoin S. (1981) Axonal transport of enzymes and labeled proteins in experimental axonopathy induced by p-bromophenylacetylurea. Brain Res. 229, 103-123.

Jakobsen J. and Sidenius P. (1983) Early and dose dependent decrease of retrograde axonal transport in acrylamide intoxicated rats. J. Neurochem. 40, 447-454.

Kuzuhara S. and Chou S. M. (1981a) Impairment of retrograde axonal transport of horseradish peroxidase (HPR) in beta,beta'iminodipropionitrile (IDPN). Neurology 31, 119.

Kuzuhara S. and Chou S. M. (1981b) Retrograde axonal transport of HRP in IDPN induced axonopathy. $J$. Neuropathol. Exp. Neurol. 40, 356.

Miller M. S. and Spencer P. S. (1984) Single doses of acrylamide reduce retrograde transport velocity. $J$. Neurochem. 43, 14011408.

Papasozomenos S. C., Autilio-Gambetti L., and Gambetti P. (1981) Reorganization of axoplasmic organelles following beta,beta'iminodipropionitrile administration. J. Cell Biol. 91, 866-871.

Papasozomenos S. C., Binder L. I., Bender P. K., and Payne M. R. (1985) Microtubule-associated protein 2 within axons of spinal motor neurons: associations with microtubules and neurofilaments in normal and beta,beta'-iminodipropionitriletreated axons. J. Cell Biol. 100, 74-85.

Sahenk Z. and Mendell J. R. (1980) Axoplasmic transport in zinc pyridinethione neuropathy: evidence for an abnormality in distal turnaround. Brain Res. 86, 343-353.

Sahenk Z. and Mendell J. R. (1981) Acrylamide and 2,5 hexanedione neuropathies: abnormal bidirectional transport rate in distal axons. Brain Res, 219, 397-405. 